Contrib. Plasma Phys. 39 (1999) 329,

\title{
Quantum Kinetic Theory for Laser Plasmas. Dynamical Screening in Strong Fields
}

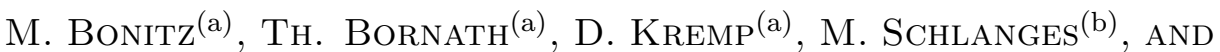 \\ W.D. KRAEFT ${ }^{(b)}$
}

(a) Universität Rostock, Fachbereich Physik, Universitätsplatz 3, D-18051 Rostock

(b) Ernst-Moritz-Arndt Universität Greifswald, Institut für Physik,

Domstr. 10a, 17487 Greifswald, Germany

\begin{abstract}
A quantum kinetic theory for correlated charged-particle systems in strong time-dependent electromagnetic fields is developed. Our approach is based on a systematic gauge-invariant nonequilibrium Green's functions formulation. Extending our previous analysis [1] we concentrate on the selfconsistent treatment of dynamical screening and electromagnetic fields which is applicable to arbitrary nonequilibrium situations. The resulting kinetic equation generalizes previous results to quantum plasmas with full dynamical screening and includes many-body effects. It is, in particular, applicable to the interaction of dense plasmas with strong electromagnetic fields, including laser fields and x-rays. Furthermore, results for the modification of the plasma screening and the longitudinal field fluctuations due to the electromagnetic field are presented.
\end{abstract}

\section{Introduction}

With the progress in short-pulse laser technology [2] high intensity electromagnetic fields are becoming broadly available. In particular, they make it possible to create strongly correlated quantum plasmas under extreme nonequilibrium conditions which opens a broad range of applications, e.g. [3]. At the same time, optical techniques for time-resolved diagnostics are improving remarkably «4. These developments create the need for a quantum kinetic theory of dense nonideal plasmas in intense laser fields.

Nonequilibrium properties of dense plasmas in which collisions are important are usually studied on the basis of kinetic equations of the Boltzmann type. However, in spite of their fundamental character, Boltzmann-like kinetic equations have a number of shortcomings, in particular in view of their application to dense plasmas in intense laser fields:

i) they are valid only for times larger than the correlation (or collision) time $\tau_{\text {corr }} \sim \omega_{p l}^{-1}$,

ii) they conserve only the mean kinetic energy instead of the sum of kinetic and potential energy,

iii) they are valid only in the weak field limit since the corresponding collision integrals are independent of the electromagnetic field,

iv) they are not applicable to high-frequency processes (fields), where $\omega>\omega_{p l}$, cf. i).

Obviously, in the case of strong correlations, high-frequency electromagnetic fields and/or short-time phenomena generalizations are necessary.

Generalized kinetic equations for correlated plasmas have been derived already in the 60ies by Prigogine [5], Zwanzig [6], Kadanoff and Baym [7, 8], Balescu [9, 10], Silin [11], Klimontovich [12, 13, 14] and others. In recent years, the increasing interest in ultrafast processes has revived the theoretical activities, e.g. [15, 16, 17, 18], acompanied by progress in numerical solutions, e.g. [15, 19, 20]; for textbook overviews, see [21, 22]. Furthermore, 
kinetic equations for classical plasmas in high-frequency fields have been derived for the first time in papers of Silin [23, 24, 25]. Among other problems, he computed the highfrequency conductivity of a plasma. We mention that, for the weak-field limit, this problem has been studied by many authors, including Oberman et al. 26, 27] and DuBois et al. [28]. An essential further development of the theory has been given by Klimontovich and co-workers 29, 14. Klimontovich used his powerful technique of second quantization in phase space [30, 12] to investigate the density-density and microfield fluctuations in low and high-frequency fields. This allowed him to derive collision integrals for classical plasmas in strong fields which take into account dynamical screening and to derive a complete theory of transport processes [29, 31]. A result of central importance is an expression for the collisional heating rate and the electron-ion collision frequency in strong fields in terms of the imaginary part of the inverse dielectric function $\operatorname{Im} \epsilon^{-1}$ [14]. Recently, expressions of the same form were derived again [32]. For a recent overview on the collision frequency in laser plasmas, we refer to Mulser et al. [33, 34].

The above kinetic theories for plasmas in electromagnetic fields were limited to classical plasmas. A first extension to quantum plasmas was given by Silin and Uryupin [35]. More recently, a kinetic equation for dense quantum plasmas in strong static fields has been derived [36, 37], whereas a systematic quantum kinetic theory for plasmas in strong fields of arbitrary time dependence was presented in ref. [1]. There, electron-electron and electronion quantum collision integrals were derived within the static Born approximation (Landau collision integrals).

In this paper, we extend this theory to the case of full dynamical screening for dense quantum plasmas under arbitrary nonequilibrium conditions by using the random phase approximation (polarization approximation) for the particle-particle scattering processes. Our approach is based on the nonequilibrium Green's functions formalism which allows for the most straightforward derivation and for an explicit solution of the gauge problem. We derive a kinetic equation which is a generalization of Klimontovich's classical result 229 and, on the other hand, generalizes previous quantum results for the case of zero field [38, 39] and static field [40]. Furthermore, we derive results for the polarization and screening properties and longitudinal field fluctuations in a strong field of arbitrary time-dependence.

\section{Basic physical problems and definitions}

We consider the time evolution of a dense charged particle system under the influence of a strong time-dependent electromagnetic field and inter-particle correlations.

\subsection{Free particle motion}

It is instructive to recall first the motion of classical free charges in an external field. From integrating Newton's equation, $m_{a} d \mathbf{v}_{a} / d t=e_{a} \mathbf{E}(t)$, we obtain the velocity change of a particle with charge $e_{a}$ and mass $m_{a}$ in the field $\mathbf{E}(t)$ during a time interval $\left[t^{\prime}, t\right]$,

$$
\Delta \mathbf{v}_{a}\left(t, t^{\prime}\right)=\frac{e_{a}}{m_{a}} \int_{t^{\prime}}^{t} d \bar{t} \mathbf{E}(\bar{t})
$$

and the field induced displacement

$$
\Delta \mathbf{r}_{a}\left(t, t^{\prime}\right)=\frac{e_{a}}{m_{a}} \int_{t^{\prime}}^{t} d \tilde{t} \int_{t^{\prime}}^{\tilde{t}} d \bar{t} \mathbf{E}(\bar{t})=\frac{e_{a}}{m_{a}} \int_{t^{\prime}}^{t} d \tilde{t} \int_{\tilde{t}}^{t} d \bar{t} \mathbf{E}(\bar{t}) .
$$

In the equations above, we have dropped contributions from the acceleration and velocity at the initial moment $t^{\prime}$ since they are not related to the field. A further important quantity is the average kinetic energy

$$
\overline{E_{a}^{\mathrm{kin}}}=\frac{m_{a}}{2} \frac{1}{T} \int_{0}^{T} d t v_{a}^{2}(t),
$$


where, in case of a periodic field, $T$ is the oscillation period. Below we will also need the change of the relative velocity of a particle pair $a, b$ gained in the field $E(t)$

$$
\Delta \mathbf{v}_{a b}\left(t, t^{\prime}\right) \equiv \Delta \mathbf{v}_{a}\left(t, t^{\prime}\right)-\Delta \mathbf{v}_{b}\left(t, t^{\prime}\right)=\left(\frac{e_{a}}{m_{a}}-\frac{e_{b}}{m_{b}}\right) \int_{t^{\prime}}^{t} d \bar{t} \mathbf{E}(\bar{t}),
$$

and the change of the inter-particle distance $\Delta \mathbf{r}_{a b}$

$$
\Delta \mathbf{r}_{a b}\left(t, t^{\prime}\right) \equiv \Delta \mathbf{r}_{a}\left(t, t^{\prime}\right)-\Delta \mathbf{r}_{b}\left(t, t^{\prime}\right)=\left(\frac{e_{a}}{m_{a}}-\frac{e_{b}}{m_{b}}\right) \int_{t^{\prime}}^{t} d \tilde{t} \int_{\tilde{t}}^{t} d \bar{t} \mathbf{E}(\bar{t})
$$

The most important special case is that of a harmonic time dependence

$$
\mathbf{E}(t)=\mathbf{E}_{0} \cos \Omega t,
$$

which leads to the following explicit results for the quantities introduced above

$$
\begin{aligned}
\Delta \mathbf{v}_{a}\left(t, t^{\prime}\right) & =\mathbf{v}_{a}^{0}\left[\sin \Omega t-\sin \Omega t^{\prime}\right], \\
\Delta \mathbf{r}_{a}\left(t, t^{\prime}\right) & =\mathbf{r}_{a}^{0}\left[\Omega\left(t-t^{\prime}\right) \sin \Omega t+\cos \Omega t-\cos \Omega t^{\prime}\right] .
\end{aligned}
$$

Here, we introduced two important quantities, the "quiver" velocity

$$
\mathbf{v}_{a}^{0} \equiv \frac{e_{a} \mathbf{E}_{0}}{m_{a} \Omega}
$$

and the so-called excursion amplitude

$$
\mathbf{r}_{a}^{0} \equiv \frac{e_{a} \mathbf{E}_{0}}{m_{a} \Omega^{2}}
$$

Furthermore, the cycle averaged $(T=2 \pi / \Omega)$ kinetic energy gain of a charged particle (3) is the so-called ponderomotive energy

$$
\overline{E_{a}^{\mathrm{kin}}}=\varepsilon_{a}^{\mathrm{pond}}=\frac{e_{a}^{2} E_{0}^{2}}{4 m_{a} \Omega^{2}} .
$$

Obviously, the above results may be extended to electromagnetic fields with arbitrary time dependence, for example by expanding the field in terms of harmonic components. Nevertheless, it is useful to explicitly consider a second situation frequently encountered in modern applications: pulsed fields, e.g. those produced by femtosecond lasers. We will consider pulses of the following form

$$
\mathbf{E}(t)=\mathbf{E}_{p}(t) \cos \Omega t, \quad \mathbf{E}_{p}(t)=2 \mathbf{E}_{0} \sin \Omega_{p} t, \quad 0 \leq \Omega_{p} t \leq \pi,
$$

and $E_{p} \equiv 0$ otherwise. Typically, $\Omega_{p} \ll \Omega$, although modern femtosecond laser pulses may be as short as a few periods of the main frequency $\Omega$. For the field (12), we obtain the velocity change and displacement

$$
\begin{aligned}
\Delta \mathbf{v}_{a}\left(t, t^{\prime}\right) & =-\sum_{s= \pm} \mathbf{v}_{a}^{0 s}\left[\sin \Omega^{s} t-\sin \Omega^{s} t^{\prime}\right], \quad \mathbf{v}_{a}^{0 \pm} \equiv \frac{e_{a} \mathbf{E}_{0}}{m_{a} \Omega^{ \pm}}, \quad \Omega^{ \pm} \equiv \Omega_{p} \pm \Omega \\
\Delta \mathbf{r}_{a}\left(t, t^{\prime}\right) & =\sum_{s= \pm} \mathbf{r}_{a}^{0 s}\left[-\Omega^{s}\left(t-t^{\prime}\right) \cos \Omega^{s} t+\sin \Omega^{s} t-\sin \Omega^{s} t^{\prime}\right]
\end{aligned}
$$

with $\mathbf{r}_{a}^{0 \pm} \equiv e_{a} \mathbf{E}_{0} / m_{a} \Omega^{ \pm 2}$, whereas the change of relative velocity and two-particle distance, $\Delta \mathbf{v}_{a b}$ and $\Delta \mathbf{r}_{a b}$ follow from $\Delta \mathbf{v}_{a}$ and $\Delta \mathbf{r}_{a}$ by replacing $\mathbf{v}_{a}^{0 \pm}$ by $\mathbf{v}_{a}^{0 \pm}-\mathbf{v}_{b}^{0 \pm}$ and $\mathbf{r}_{a}^{0 \pm}$ by $\mathbf{r}_{a}^{0 \pm}-\mathbf{r}_{b}^{0 \pm}$, respectively. 
The above results trivially include the case of a time-independent electric field which is recovered by letting in Eq. (6) $\Omega \rightarrow 0$. The corresponding results are

$$
\Delta \mathbf{v}_{a}\left(t, t^{\prime}\right)=\frac{e_{a}}{m_{a}} \mathbf{E}_{0}\left(t-t^{\prime}\right), \quad \Delta \mathbf{r}_{a}\left(t, t^{\prime}\right)=\frac{1}{2} \frac{e_{a}}{m_{a}} \mathbf{E}_{0}\left(t-t^{\prime}\right)^{2}
$$

It is instructive to consider a number of parameters which characterize the state of the plasma, field strength, quantum properties etc:

1. The field strength can be characterized by the ratio $\alpha=v_{a}^{0} / v_{\mathrm{th}, a}$ of the amplitude of the oscillation velocity (quiver velocity) $v_{a}^{0}$, Eq. (7), to the thermal velocity $v_{\mathrm{th}, a}=$ $\left(k T / m_{a}\right)^{1 / 2}$.

2. The relative importance of the field and of particle-particle interaction is characterized by $\beta=r_{a}^{0} / r_{D}$, where $r_{a}^{0}$ is the amplitude of the field induced displacement (8), and $r_{D}$ is the Debye radius (interaction range).

3. The frequency of the field has to be compared to the eigenfrequencies of the plasma, most importrantly, the electron Langmuir (plasma) frequency, $\gamma=\Omega / \omega_{p l}$, which reflects competition between field frequency and plasma density effects.

4. The relevance of collisional processes depends on the ratio $\delta=\nu / \Omega$, where $\nu$ is the total collision frequency of electrons in the plasma.

5. The photon energy is characterized by its ratio to the thermal energy, $\hbar \Omega / k T$.

Modern lasers easily produce strong fields which satisfy the inequalities $\alpha \gg 1$ and $\beta \gg 1$. In high-frequency fields and/or plasmas of moderate density, $\delta \ll 1$, which allows to treat collisions perturbatively, see below.

\subsection{Two-particle scattering}

Coulomb interaction between the charged carriers as well as quantum effects, obviously, may drastically modify the free particle behavior. Scattering of two particles with charges $e_{a}, e_{b}$ in quantum states $\left|\mathbf{k}_{1}\right\rangle$ and $\left|\mathbf{k}_{2}\right\rangle$ on the Coulomb potential

$$
V_{a b}(q)=\frac{4 \pi e_{a} e_{b} \hbar^{2}}{q^{2}}
$$

leads to a transfer of momentum $\mathbf{q}$ between them, so after the collision time $t \sim t_{\text {coll }}$ the particles are in momentum states $\left|\mathbf{k}_{1}+\mathbf{q}\right\rangle$ and $\left|\mathbf{k}_{2}-\mathbf{q}\right\rangle$. While conventional kinetic approaches treat collision as instantaneous, $t_{\text {coll }} \rightarrow 0$, this is not appropriate for correlated plasmas as well as in the presence of rapidly varying fields with $\Omega \cdot t_{\text {coll }}$ not being small. In this case, during the collision time, the scattering partners will be accelerated by the external field which is called intra-collisional field effect, which essentially modifies the scattering process. Using a quantum language, during the collision time, the particles may absorb photons of the electromagnetic field which is the familiar inverse bremsstrahlung, or re-emit them (bremsstrahlung).

The kinetic treatment of two-particle scattering on the Coulomb potential (13) leads to the well-known divergencies at short and long wavelengths. While the first is naturally cured by a quantum theoretical approach, the origin of the latter is the long range of the Coulomb interaction. The familiar solution lies in the replacement of the bare Coulomb potential (13) by a screened one

$$
V_{a b}(q) \rightarrow V_{a b}^{s}(\omega, q)=\frac{V_{a b}(q)}{\epsilon^{R}(\omega, q)},
$$


where $\epsilon^{R}$ is the retarded dielectric function. This is not only of fundamental interest but has also practical relevance. The dielectric function includes collective plasma oscillations and instabilities which, especially in nonequilibrium situations, may strongly enhance scattering, transport and energy exchange with the electromagnetic field (anomalous transport).

The simplest approximation for the dielectric function is the random phase approximation (RPA) being the quantum generalization of the Vlasov dielectric function

$$
\begin{aligned}
\epsilon^{R}(\omega, q ; t) & =1-\sum_{a} V_{a a}(q) \Pi_{a}^{R}(\omega, q ; t) \\
\Pi_{a}^{R}(\omega, q ; t) & =\frac{1}{\hbar} \int \frac{d^{3} k}{(2 \pi \hbar)^{3}} \frac{f_{a}(\mathbf{k} ; t)-f_{a}(\mathbf{k}+\mathbf{q} ; t)}{\hbar \omega+i \delta+\epsilon_{a}(\mathbf{k})-\epsilon_{a}(\mathbf{k}+\mathbf{q})},
\end{aligned}
$$

where $\epsilon_{a}$ denotes the single-particle energy. Interestingly, this result was derived by Klimontovich and Silin [41, 42] two years before Lindhard [43]. While this approximation is applicable to nonequilibrium situations in which the Wigner distributions $f_{a}$ are weakly timedependent (when the time scale $t$ is much longer than $2 \pi / \omega$ ), on short times or for fast processes, such as in high frequency fields, generalizations are necessary. Such generalizations avoid the assumption of separation of the two time scales and lead to an explicit dependence of the functions $\Pi, \epsilon$ and $V^{s}$ on two times. Moreover, the presence of an electromagnetic field may be expected to modify the dielectric and screening properties, leading to a very complex problem of coupled particle, screening and field dynamics. The appropriate theoretical concept to tackle this problem is provided by quantum field theory.

\section{Quantum field theoretical approach to the dynamics of plasmas in electromagnetic fields}

Numerous concepts have been developed to describe the mentioned above dynamics of particles and fields. Among them, the most systematic and powerful is the theory of nonequilibrium Green's functions. It is based on the method of relativistic quantum field theory, where charged particles and the longitudinal and transverse electromagnetic field are described on equal footing by field operators [44, 45]. From the equations of motion for the field operators - the Dirac equation and Maxwell's equations, one can derive equations of motion for all quantities of interest. Among them, the most important are two-time correlation functions (Green's functions) which allow for systematic and far-reaching generalizations of traditional kinetic theory. In this paper, we focus on nonrelativistic particle dynamics and start our derivations from the familiar equations of motion for the particle correlation functions $g^{>}$and $g^{<}$while the electromagnetic field is treated classically.

\subsection{Kadanoff-Baym Equations}

The field theoretical description of plasmas is based on the creation and annihilation operators $\psi^{\dagger}$ and $\psi$ 46] which are defined to guarantee the spin statistics theorem,

$$
\begin{aligned}
\psi_{a}(1) \psi_{b}(2) \mp \psi_{b}(2) \psi_{a}(1) & =\psi_{a}^{\dagger}(1) \psi_{b}^{\dagger}(2) \mp \psi_{b}^{\dagger}(2) \psi_{a}^{\dagger}(1)=0, \\
\psi_{a}(1) \psi_{b}^{\dagger}(2) \mp \psi_{b}^{\dagger}(2) \psi_{a}(1) & =\delta(1-2) \delta_{a, b},
\end{aligned}
$$

where $t_{1}=t_{2}$ has been assumed. The upper (lower) sign refers to bosons (fermions), $1 \equiv\left(\mathbf{r}_{1}, t_{1}, s_{1}^{3}\right)$, and $a$ labels the particle species. Below, we will drop the spin index and assume fermions. The nonequilibrium state of a correlated plasma is described by the twotime correlation functions which are statistical averages (with the initial density operator of the system) of field operator products

$$
g_{a}^{>}\left(1,1^{\prime}\right)=\frac{1}{i \hbar}\left\langle\psi_{a}(1) \psi_{a}^{\dagger}\left(1^{\prime}\right)\right\rangle, \quad g_{a}^{<}\left(1,1^{\prime}\right)=-\frac{1}{i \hbar}\left\langle\psi_{a}^{\dagger}\left(1^{\prime}\right) \psi_{a}(1)\right\rangle
$$


where $g_{a}^{>}$and $g_{a}^{<}$are, in nonequilibrium, independent from one another. They contain the complete dynamical and statistical information. The latter follows from their elements along the time diagonal: the one-particle density matrix is immediately obtained from the function $g^{<}$according to

$$
f_{a}\left(\mathbf{r}_{1}, \mathbf{r}_{1}^{\prime}, t\right)=-\left.i \hbar g_{a}^{<}\left(1,1^{\prime}\right)\right|_{t_{1}=t_{1}^{\prime}},
$$

whereas the dynamical information (e.g. the single-particle spectrum and the correlations) follows from the function values across the diagonal in the $t_{1}-t_{1}^{\prime}$-plane, in particular, from the spectral function $a\left(1,1^{\prime}\right)$,

$$
a\left(1,1^{\prime}\right) \equiv i \hbar\left\{g_{a}^{>}\left(1,1^{\prime}\right)-g_{a}^{<}\left(1,1^{\prime}\right)\right\}=i \hbar\left\{g_{a}^{R}\left(1,1^{\prime}\right)-g_{a}^{A}\left(1,1^{\prime}\right)\right\},
$$

where $g^{R / A}$ are the retarded and advanced Green's functions, defined below in Eq. (25). In the following, it will often be convenient to use microscopic and macroscopic time and space variables being defined as

$$
\begin{aligned}
& \mathbf{r}=\mathbf{r}_{1}-\mathbf{r}_{1}^{\prime}, \quad \mathbf{R}=\left(\mathbf{r}_{1}+\mathbf{r}_{1}^{\prime}\right) / 2, \\
& \tau=t_{1}-t_{1}^{\prime}, \quad t=\left(t_{1}+t_{1}^{\prime}\right) / 2 .
\end{aligned}
$$

In particular, in cases where the microscopic variables vary on much smaller scales than the macroscopic ones, it is advantageous to perform a Fourier transformation with respect to $\tau$ and/or $\mathbf{r}$ which leads to the frequency and momentum variables $\omega$ and $\mathbf{p}$, respectively. In particular, Eq. (18) then yields the familiar Wigner distribution function

$$
f_{a}(\mathbf{p}, \mathbf{R}, t)=-\left.i \hbar g_{a}^{<}\left(\mathbf{p}, \mathbf{R} ; t_{1}, t_{1}^{\prime}\right)\right|_{t_{1}=t_{1}^{\prime}=t} .
$$

The time evolution of the correlation functions in an electromagnetic field is determined by the Kadanoff-Baym equations [7, 47]

$$
\begin{aligned}
& {\left[i \hbar \frac{\partial}{\partial t_{1}}-\frac{1}{2 m_{a}}\left(\frac{\hbar}{i} \nabla_{1}-\frac{e_{a}}{c} \mathbf{A}(1)\right)^{2}-e_{a} \phi(1)\right] g_{a}^{\gtrless}\left(1,1^{\prime}\right)-\int d \overline{\mathbf{r}}_{1} \Sigma_{a}^{\mathrm{HF}}\left(1, \overline{\mathbf{r}}_{1} t_{1}\right) g_{a}^{\gtrless}\left(\overline{\mathbf{r}}_{1} t_{1}, 1^{\prime}\right)} \\
& =\int_{t_{0}}^{t_{1}} d \overline{1}\left[\Sigma_{a}^{>}(1, \overline{1})-\Sigma_{a}^{<}(1, \overline{1})\right] g_{a}^{\gtrless}\left(\overline{1}, 1^{\prime}\right)-\int_{t_{0}}^{t_{1}^{\prime}} d \overline{1} \Sigma_{a}^{\gtrless}(1, \overline{1})\left[g_{a}^{>}\left(\overline{1}, 1^{\prime}\right)-g_{a}^{<}\left(\overline{1}, 1^{\prime}\right)\right],(23)
\end{aligned}
$$

which have to be fulfilled together with the adjoint equations. Here, $t_{0}$ denotes the initial time where the system is assumed to be uncorrelated (otherwise, the equations have to be supplemented with an initial correlation contribution to $\Sigma_{a}$, cf. 48]). $\Sigma_{a}^{\mathrm{HF}}$ is the HartreeFock selfenergy (mean-field energy with exchange),

$$
\Sigma_{a}^{\mathrm{HF}}\left(11^{\prime}\right)=-i \hbar \delta\left(t_{1}-t_{1^{\prime}}\right) \sum_{b}\left\{\int d \mathbf{r}_{2} V_{a b}\left(\mathbf{r}_{1}-\mathbf{r}_{2}\right) g_{b}^{<}\left(22^{+}\right)-\delta_{a, b} V_{a b}\left(\mathbf{r}_{1}-\mathbf{r}_{1}^{\prime}\right) g_{b}^{<}\left(11^{\prime}\right)\right\},
$$

and $\Sigma_{a}^{\gtrless}$ are the correlation selfenergies which will be discussed below.

For the following derivations, it is useful to introduce, in addition, the retarded and advanced Green's functions

$$
g_{a}^{R / A}\left(1,1^{\prime}\right)= \pm \Theta\left[ \pm\left(t_{1}-t_{1}^{\prime}\right)\right]\left\{g_{a}^{>}\left(1,1^{\prime}\right)-g_{a}^{<}\left(1,1^{\prime}\right)\right\},
$$

which obey the simpler equations

$$
\begin{aligned}
& {\left[i \hbar \frac{\partial}{\partial t_{1}}-\frac{1}{2 m_{a}}\left(\frac{\hbar}{i} \nabla_{1}-\frac{e_{a}}{c} \mathbf{A}(1)\right)^{2}-e_{a} \phi(1)\right] g_{a}^{R / A}\left(1,1^{\prime}\right)} \\
& -\int d 2 \Sigma_{a}^{R / A}(1,2) g_{a}^{R / A}\left(2,1^{\prime}\right)=\delta\left(1-1^{\prime}\right) .
\end{aligned}
$$


In equations (23) and (26), the electromagnetic field is given by the vector and scalar potentials $\mathbf{A}$ and $\phi$ and will be treated classically. $\mathbf{A}$ is the full vector potential (external plus induced) which obeys Maxwell's equations, whereas $\phi$ is understood as to be due to external sources only, the induced longitudinal field is fully accounted for in the screened Coulomb potential $V^{s}$ which enters the selfenergies $\Sigma_{a}^{>}$and $\Sigma_{a}^{<}$, see below.

Although one can directly analyze and solve the two-time Kadanoff-Baym equations (23), e.g. [19], for an overview, see [22], it is easier to consider the kinetic equation for the Wigner distribution function (22), which we will be concerned with here. This equation is immediately obtained from the equal time limit, $t_{1}=t_{1}^{\prime}=t$, of Eq. (23) plus its adjoint. Introducing further the variables $\mathbf{R}$ and $\mathbf{r}$, Eqs. (20) and (21), we obtain, after Fourier transformation with respect to $\mathbf{r}$, for the spatially homogeneous case,

$$
\frac{\partial}{\partial t} f_{a}(\mathbf{p}, t)=-2 \operatorname{Re} \int_{t_{0}}^{t} d \bar{t}\left\{\Sigma_{a}^{>}(\mathbf{p} ; t, \bar{t}) g_{a}^{<}(\mathbf{p} ; \bar{t}, t)-\Sigma_{a}^{<}(\mathbf{p} ; t, \bar{t}) g_{a}^{>}(\mathbf{p} ; \bar{t}, t)\right\} .
$$

This is an exact equation and, therefore, well suited for deriving generalized kinetic equations. However, this equation is not closed yet since it contains under the collision integral functions depending on two times. Therefore, to obtain explicit expressions for the collision integral, one has:

1. to find appropriate approximations for the self energy. For this, the Green's functions approach provides powerful approximation schemes based on Feynman diagrams which allow for a very systematic development of the theory. Here, we are interested in the plasma dynamics with screening effects properly included. Therefore, the appropriate choice for the selfenergy will be the random phase approximation (RPA);

2. to express the correlation functions $g_{a}^{\gtrless}$ as functionals of the Wigner functions $f_{a}$ (reconstruction problem). This problem can be solved approximately on the basis of the generalized Kadanoff-Baym ansatz (GKBA) of Lipavský et al. [49], see Sec. 3.3.

\subsection{Gauge-invariant Green's functions}

It is well known that the electromagnetic field can be introduced in various ways (gauges) which may lead to essentially different explicit forms of the resulting kinetic equations. Although alternative derivations are successfully applied too, gauge invariance becomes a particular problem if the resulting kinetic equations are treated by means of approximations, such as retardation or gradient expansions. A critical issue is that the result of these approximations maybe essentially different in different gauges, see e.g. 221] for examples. To avoid these difficulties, we will formulate the theory in terms of correlation functions which are made explicitly gauge-invariant. While the main results have been presented in Ref. [1], here we provide some additional details.

In this section, we use a co-variant 4-vector notation as it makes the following transformations more compact and symmetric. The corresponding definitions are

$$
A_{\mu}=(c \phi, \mathbf{A}), \quad x_{\mu}=(c \tau, \mathbf{r}), \quad X_{\mu}=(c t, \mathbf{R}),
$$

and the convention $a_{\mu} b^{\mu}=a_{0} b_{0}-\mathbf{a b}$ is being used.

One readily proofs that the Kadanoff-Baym equations (23) remain covariant under gauge transformations, i.e., under the following transformations of the potentials and field operators

$$
A_{\mu}^{\prime}(x)=A_{\mu}(x)-\partial_{\mu} \chi(x) \quad \psi_{a}^{\prime}(x)=e^{\frac{i}{\hbar} \frac{e a}{c} \chi(x)} \psi_{a}(x),
$$

The corresponding gauge transform of the Green's functions leads to

$$
g_{a}^{\prime}(x, X)=e^{\frac{i}{\hbar} \frac{e_{a}}{c}\left[\chi\left(X+\frac{x}{2}\right)-\chi\left(X-\frac{x}{2}\right)\right]} g_{a}(x, X) .
$$


Following an idea of Fujita [50], we now introduce a gauge-invariant Green's function $g(\mathbf{k}, X)$ which is given by the modified Fourier transform

$$
g_{a}(\mathbf{k}, X)=\int \frac{d^{4} x}{(2 \pi)^{4}} \exp \left\{i \int_{-\frac{1}{2}}^{\frac{1}{2}} d \lambda x_{\mu}\left[k^{\mu}+\frac{e_{a}}{c} A^{\mu}(X+\lambda x)\right]\right\} g_{a}(x, X),
$$

where use has been made of the identity

$$
\chi\left(X+\frac{x}{2}\right)-\chi\left(X-\frac{x}{2}\right)=\int_{-\frac{1}{2}}^{\frac{1}{2}} d \lambda \frac{d}{d \lambda} \chi(X+\lambda x)=x_{\mu} \partial^{\mu} \int_{-\frac{1}{2}}^{\frac{1}{2}} d \lambda \chi(X+\lambda x) .
$$

Indeed, one readily confirms that under any gauge transform (28), the phase factors cancel, and $g^{\prime}(\mathbf{k}, X) \equiv g(\mathbf{k}, X), 21$.

In the following, we focus on spatially homogeneous electric fields and use the vector potential gauge

$$
A_{0}=\phi=0 ; \quad \mathbf{A}=-c \int_{-\infty}^{t} d \bar{t} \mathbf{E}(\bar{t})
$$

In this case, relation (29) simplifies to

$$
g_{a}(\mathbf{k}, \omega ; \mathbf{R}, t)=\int d \tau d \mathbf{r} \exp \left[i \omega \tau-\frac{i}{\hbar} \mathbf{r} \cdot\left(\mathbf{k}+\frac{e_{a}}{c} \int_{t-\frac{\tau}{2}}^{t+\frac{\tau}{2}} \frac{d t^{\prime}}{\tau} \mathbf{A}\left(t^{\prime}\right)\right)\right] g_{a}(\mathbf{r}, \tau ; \mathbf{R}, t),
$$

what means that the gauge-invariant Green's function $g(\mathbf{k})$ follows from the Wigner transformed function $g_{a}(\mathbf{p})$ by replacing the canonical momentum $\mathbf{p}$ by the gauge-invariant kinematic momentum $\mathbf{k}$ according to

$$
\mathbf{p}=\mathbf{k}+\frac{e_{a}}{c} \int_{t-\frac{\tau}{2}}^{t+\frac{\tau}{2}} d t^{\prime} \frac{\mathbf{A}\left(t^{\prime}\right)}{\tau}
$$

Let us illustrate this for the examples studied in Sec. 2. For a harmonic electric field given by Eq. (6), the vector potential and the momentum relation become, according to Eq. (30),

$$
\mathbf{A}(t)=-\frac{c \mathbf{E}_{0}}{\Omega} \sin \Omega t, \quad \mathbf{p}=\mathbf{k}+\frac{2}{\tau} \frac{\mathbf{E}_{0}}{\Omega^{2}} \sin \Omega t \sin \frac{\Omega \tau}{2} .
$$

Similarly, for a pulsed field of the form Eq. (12), the result for the vector potential and the momenta is

$$
\mathbf{A}(t)=c \mathbf{E}_{0} \sum_{s= \pm} \frac{\cos \Omega^{s} t}{\Omega^{s}}, \quad \mathbf{p}=\mathbf{k}+\frac{2 \mathbf{E}_{0}}{\tau} \sum_{s= \pm} \frac{1}{\Omega^{s}} \cos \Omega^{s} t \sin \frac{\Omega^{s} \tau}{2} .
$$

Finally, for a static field we obtain,

$$
\mathbf{A}(t)=-c \mathbf{E}_{0} t, \quad \mathbf{p}=\mathbf{k}+e_{a} \mathbf{E}_{0} t .
$$

For the derivations below, we will need the gauge invariant Fourier transform of the convolution of two functions which, in the homogeneous case, is given by

$$
I\left(\mathbf{r}_{1}-\mathbf{r}_{1}^{\prime} ; t_{1}, t_{1}^{\prime}\right)=\int d \bar{t} d \overline{\mathbf{r}} B\left(\mathbf{r}_{1}-\overline{\mathbf{r}} ; t_{1}, \bar{t}\right) C\left(\overline{\mathbf{r}}-\mathbf{r}_{1}^{\prime} ; \bar{t}, t_{1}^{\prime}\right) .
$$


After straightforward manipulations which involve the back transform of (31), we arrive at

$$
\begin{aligned}
& I\left(\mathbf{k} ; t_{1}, t_{1}^{\prime}\right)=\int d \bar{t} B\left[\mathbf{k}+\frac{e_{a}}{c} \int_{t_{1}^{\prime}}^{t_{1}} d t^{\prime \prime} \frac{\mathbf{A}\left(t^{\prime \prime}\right)}{t_{1}-t_{1}^{\prime}}-\frac{e_{a}}{c} \int_{\bar{t}}^{t_{1}} d t^{\prime \prime} \frac{\mathbf{A}\left(t^{\prime \prime}\right)}{t_{1}-\bar{t}} ; t_{1}, \bar{t}\right] \\
& \times C\left[\mathbf{k}+\frac{e_{a}}{c} \int_{t_{1}^{\prime}}^{t_{1}} d t^{\prime \prime} \frac{\mathbf{A}\left(t^{\prime \prime}\right)}{t_{1}-t_{1}^{\prime}}-\frac{e_{a}}{c} \int_{t_{1}^{\prime}}^{\bar{t}} d t^{\prime \prime} \frac{\mathbf{A}\left(t^{\prime \prime}\right)}{\bar{t}-t_{1}^{\prime}} ; \bar{t}, t_{1}^{\prime}\right] .
\end{aligned}
$$

In particular, the derivation of the collision integral in the kinetic equation for the Wigner function, requires the equal-time limit of this expression, $t_{1}=t_{1}^{\prime}=t$,

$$
\begin{aligned}
& I(\mathbf{k} ; t)=\int d \bar{t} B\left[\mathbf{k}+\frac{e_{a}}{c} \mathbf{A}(t)-\frac{e_{a}}{c} \int_{\bar{t}}^{t} d t^{\prime \prime} \frac{\mathbf{A}\left(t^{\prime \prime}\right)}{t-\bar{t}} ; t, \bar{t}\right] \\
& \times C\left[\mathbf{k}+\frac{e_{a}}{c} \mathbf{A}(t)-\frac{e_{a}}{c} \int_{\bar{t}}^{t} d t^{\prime \prime} \frac{\mathbf{A}\left(t^{\prime \prime}\right)}{t-\bar{t}} ; \bar{t}, t\right],
\end{aligned}
$$

where $B$ and $C$ will be replaced by $g \gtrless$ and $\Sigma \gtrless$. Notice that in this case the momentum arguments of $B$ and $C$ are equal. To simplify the notation below, we introduce the field induced momentum shift

$$
\mathbf{K}_{a}^{A}\left(t, t^{\prime}\right) \equiv \frac{e_{a}}{c} \int_{t^{\prime}}^{t} d t^{\prime \prime} \frac{\mathbf{A}(t)-\mathbf{A}\left(t^{\prime \prime}\right)}{t-t^{\prime}},
$$

which has the important property

$$
\mathbf{K}_{a}^{A}\left(t, t^{\prime}\right)-\mathbf{K}_{a}^{A}\left(t^{\prime}, t\right)=\frac{e_{a}}{c}\left\{\mathbf{A}(t)-\mathbf{A}\left(t^{\prime}\right)\right\}=-e_{a} \int_{t^{\prime}}^{t} d t^{\prime \prime} \mathbf{E}\left(t^{\prime \prime}\right) \equiv \mathbf{Q}_{a}\left(t, t^{\prime}\right),
$$

where $\mathbf{Q}_{a}$ is nothing but minus the momentum gain of a free particle in the field, $\mathbf{Q}_{a}\left(t, t^{\prime}\right)=$ $-m_{a} \Delta \mathbf{v}_{a}\left(t, t^{\prime}\right)$, cf. Eq. (11). Another important relation follows from multiplication by the time interval:

$$
\frac{1}{m_{a}} \mathbf{K}_{a}^{A}\left(t, t^{\prime}\right) \cdot\left(t-t^{\prime}\right) \equiv-\mathbf{R}_{a}\left(t, t^{\prime}\right)
$$

where $\mathbf{R}_{a}$ is just the field induced displacement of a free particle, Eq. (2)), i.e. $\mathbf{R}_{a}\left(t, t^{\prime}\right)=$ $\Delta \mathbf{r}_{a}\left(t, t^{\prime}\right)$.

Definition (37) allows us to rewrite Eq. (36) as

$$
I(\mathbf{k} ; t)=\int d \bar{t} B\left[\mathbf{k}+\mathbf{K}_{a}^{A}(t, \bar{t}) ; t, \bar{t}\right] C\left[\mathbf{k}+\mathbf{K}_{a}^{A}(t, \bar{t}) ; t, \bar{t}\right] .
$$

\subsection{Gauge invariant propagator. Generalized Kadanoff-Baym ansatz}

As noted in Sec. 3.1, for the derivation of the collision integral in the kinetic equation, we need to express the functions $g^{>}$and $g^{<}$in terms of the Wigner function. In addition, such a reconstruction ansatz involves the retarded and advanced Green's functions $g^{R / A}\left(t, t^{\prime}\right)$ for which suitable expressions have to be found. We first determine these quantities for free particles in an electromagnetic field which allows to simplify Eq. (26) to

$$
\left[i \hbar \frac{\partial}{\partial t_{1}}-\frac{1}{2 m_{a}}\left\{\mathbf{p}-\frac{e_{a}}{c} \mathbf{A}\left(t_{1}\right)\right\}^{2}\right] g_{a}^{R / A}\left(\mathbf{p} ; t_{1}, t_{1}^{\prime}\right)=\delta\left(t_{1}-t_{1}^{\prime}\right)
$$


which is solved immediately by

$$
g_{a}^{R}(\mathbf{p} ; \tau, t)=-\frac{i}{\hbar} \Theta(\tau) \exp \left[-\frac{i}{\hbar} \int_{t-\frac{\tau}{2}}^{t+\frac{\tau}{2}} d t^{\prime}\left[\mathbf{p}-\frac{e_{a}}{c} \mathbf{A}\left(t^{\prime}\right)\right]^{2} / 2 m_{a}\right],
$$

and $g_{a}^{A}$ is obtained from the symmetry relation $g_{a}^{A}(\mathbf{p} ; \tau, t)=\left[g_{a}^{R}(\mathbf{p} ;-\tau, t)\right]^{*}$. From this result, we can calculate the spectral function $a\left(t, t^{\prime}\right)$, Eq. (19),

$$
a_{a}(\mathbf{p} ; \tau, t)=\exp \left[-\frac{i}{\hbar} \int_{t-\frac{\tau}{2}}^{t+\frac{\tau}{2}} d t^{\prime}\left[\mathbf{p}-\frac{e_{a}}{c} \mathbf{A}\left(t^{\prime}\right)\right]^{2} / 2 m_{a}\right] .
$$

Obviously, the results (41) and (42) are gauge-dependent since $g_{a}^{R / A}$ and $a_{a}$ are functions of the canonic momentum p. But one can easily obtain the corresponding gauge-invariant results by applying the transform (29), with the result

$$
\begin{aligned}
g_{a}^{R}(\mathbf{k} ; \tau, t) & =-\frac{i}{\hbar} \Theta(\tau) e^{-\frac{i}{\hbar}\left[\frac{k^{2}}{2 m_{a}} \tau+S_{a}(\mathbf{A} ; \tau, t)\right]} \\
\text { where } S_{a}(\mathbf{A} ; \tau, t) & =\frac{e_{a}^{2}}{2 m_{a} c^{2}}\left[\int_{t-\frac{\tau}{2}}^{t+\frac{\tau}{2}} d t^{\prime} A^{2}\left(t^{\prime}\right)+\frac{1}{\tau}\left(\int_{t-\frac{\tau}{2}}^{t+\frac{\tau}{2}} d t^{\prime} \mathbf{A}\left(t^{\prime}\right)\right)^{2}\right] .
\end{aligned}
$$

This result has a simple physical interpretation. For a free particle without field, the spectral function shows free undamped oscillations along $\tau$ (i.e. perpendicular to the time diagonal) with the one-particle energy $\epsilon_{a}(k)=k^{2} / 2 m_{a}$, and its Fourier transform is

$$
a_{a}^{\text {free }}(\mathbf{k} ; \omega, t)=\delta\left[\hbar \omega-\epsilon_{a}(k)\right] .
$$

This clearly underlines the meaning of the functions $g^{R / A}$ and, in particular, the spectral function $a$ - they contain the full information on the single-particle energy spectrum. Furthermore, in a correlated system, the single-particle spectrum is affected by interactions with other particles. This leads to a shift of the oscillation frequency and to damping of the oscillations, i.e. to finite life time effects, and it is reasonable to call the corresponding single-particle excitations quasi-particles. On the other hand, the result (43) reflects the influence of an electromagnetic field on the particle spectrum, while correlation effects have been neglected. Eq. (43) shows that the field causes a time-dependent shift of the singleparticle energy which, obviously, reflects the well-known fact that the proper eigenstates of the system contain the electromagnetic field and are given by Volkov states [51]. The spectrum may even contain additional peaks which becomes particularly transparent in the limiting case of a harmonic time dependence: For the field (6), the time integrations in $S$ can be performed, and simple trigonometric relations lead to 52.

$$
S_{a}(\mathbf{A} ; \tau, t)=\varepsilon_{a}^{\text {pond }} \tau\left[1-\frac{\sin \Omega \tau \cos 2 \Omega t}{\Omega \tau}+\frac{8 \sin ^{2} \Omega t \sin ^{2} \frac{\Omega \tau}{2}}{(\Omega \tau)^{2}}\right],
$$

where $\varepsilon_{a}^{\text {pond }}$ is the ponderomotive potential which was introduced in Eq. (11). The first term in the brackets leads to a shift of the single-particle energy, the average kinetic energy of the particles increased by $\varepsilon_{a}^{\text {pond }}$. The remaining terms modify the spectrum qualitatively giving rise to additional peaks which are related to photon sidebands [52]. 
Now we turn to the solution of the reconstruction problem. The simplest solution is the common Kadanoff-Baym ansatz,

$$
\pm i \hbar g_{a}^{\gtrless}(\mathbf{p} ; \omega, t)=a_{a}^{\text {free }}(\mathbf{p} ; \omega, t) f \gtrless(\mathbf{p} ; t),
$$

where $f^{<} \equiv f$ and $f^{>} \equiv 1-f$, and the upper (lower) sign refers to $g^{>}\left(g^{<}\right)$. Indeed, the two-time functions $g_{a}^{>}\left(t_{1}, t_{1}^{\prime}\right)$ and $g_{a}^{<}\left(t_{1}, t_{1}^{\prime}\right)$ are now expressed in terms of one-time Wigner distribution functions and a known spectral function. However, due to the expected retardation effects, this ansatz is not applicable here. As mentioned above, a more general solution which properly takes into account retardation (memory) effects is the generalized Kadanoff-Baym ansatz proposed by Lipavský et al. [49] which reads

$$
g_{a}^{\gtrless}\left(\mathbf{p} ; t_{1}, t_{1}^{\prime}\right)=i \hbar g_{a}^{R}\left(\mathbf{p} ; t_{1}, t_{1}^{\prime}\right) g_{a}^{\gtrless}\left(\mathbf{p} ; t_{1}^{\prime}, t_{1}^{\prime}\right)-i \hbar g_{a}^{\gtrless}\left(\mathbf{p} ; t_{1}, t_{1}\right) g_{a}^{A}\left(\mathbf{p} ; t_{1}, t_{1}^{\prime}\right),
$$

where for the functions on the time diagonal $\pm i \hbar g_{a}^{\gtrless}\left(\mathbf{p} ; t_{1}, t_{1}\right)=f \gtrless\left(\mathbf{p} ; t_{1}\right)$, cf. Eq. (22). Within the quasiparticle approximation and with static selfenergies, Eq. (48) is exact. In more complex situations, it is an approximation to the exact reconstruction solution, which has prooved extremely successful in many applications. In particular, it has been used for more general selfenergies and also with more general propagators $g_{a}^{R / A}$, e.g. [21]. We, therefore will use this ansatz below.

Eq. (48) is written in terms of the momentum $\mathbf{p}$ and is, therefore, gauge-dependent. To transform this relation into a gauge-invariant form, we use its coordinate representation,

$$
\begin{aligned}
\pm g_{a}^{\gtrless}\left(\mathbf{r}_{1}-\mathbf{r}_{1}^{\prime} ; t_{1}, t_{1}^{\prime}\right) & =\int d \overline{\mathbf{r}} g_{a}^{R}\left(\mathbf{r}_{1}-\overline{\mathbf{r}} ; t_{1}, t_{1}^{\prime}\right) f_{a}^{\gtrless}\left(\overline{\mathbf{r}}-\mathbf{r}_{1}^{\prime} ; t_{1}^{\prime}\right) \\
& -\int d \overline{\mathbf{r}} f_{a}^{\gtrless}\left(\mathbf{r}_{1}-\overline{\mathbf{r}} ; t_{1}\right) g_{a}^{A}\left(\overline{\mathbf{r}}-\mathbf{r}_{1}^{\prime} ; t_{1}, t_{1}^{\prime}\right),
\end{aligned}
$$

and apply the gauge-invariant Fourier transform (31) together with the back transforms of $g_{a}^{R / A}$ and $f_{a}^{\gtrless}$ which leads to the gauge-invariant generalization of the GKBA

$$
\pm g_{a}^{\gtrless}\left(\mathbf{k} ; t_{1}, t_{1}^{\prime}\right)=g_{a}^{R}\left(\mathbf{k} ; t_{1}, t_{1}^{\prime}\right) f_{a}^{\gtrless}\left[\mathbf{k}-\mathbf{K}_{a}^{A}\left(t^{\prime}, t\right) ; t_{1}^{\prime}\right]-f_{a}^{\gtrless}\left[\mathbf{k}-\mathbf{K}_{a}^{A}\left(t, t^{\prime}\right) ; t_{1}\right] g_{a}^{A}\left(\mathbf{k} ; t_{1}, t_{1}^{\prime}\right),
$$

where the definition (37) for $\mathbf{K}_{a}^{A}$ has been used. As in the field-free case, the first term is nonzero only for $t_{1} \geq t_{1}^{\prime}$ and the second in the opposite case. Notice the difference of the time arguments in the two distribution functions.

\section{General kinetic equation for quantum particles including screening and electromagnetic fields}

We now come back to the time-diagonal limit of the Kadanoff-Baym equations, cf. Eqs. (23) and (27), and derive the quantum kinetic equation for a plasma in a laser field thereby fully taking into account dynamical screening. Again, it is advantageous to derive this equation for the gauge-invariant Wigner distribution. To this end, we take the Fourier transform (31) of the time-diagonal Kadanoff-Baym equation

$$
\frac{\partial}{\partial t} f_{a}\left(\mathbf{k}_{a}, t\right)+e_{a} \mathbf{E}(t) \cdot \nabla_{\mathbf{k}} f_{a}\left(\mathbf{k}_{a}, t\right)=-2 \operatorname{Re} \int_{t_{0}}^{t} d \bar{t}\left\{\Sigma_{a}^{>} g_{a}^{<}-\Sigma_{a}^{<} g_{a}^{>}\right\}=I_{a}\left(\mathbf{k}_{a}, t\right),
$$

where the full arguments are, according to the convolution relation (40), given by

$$
\Sigma_{a}^{\gtrless} g_{a}^{\lessgtr} \equiv \Sigma_{a}^{\gtrless}\left[\mathbf{k}_{a}+\mathbf{K}_{a}^{A}(t, \bar{t}) ; t, \bar{t}\right] g_{a}^{\lessgtr}\left[\mathbf{k}_{a}+\mathbf{K}_{a}^{A}(t, \bar{t}) ; \bar{t}, t\right] .
$$

This expression is valid for arbitrary approximations for the selfenergies $\Sigma^{>}$and $\Sigma^{<}$. In our previous paper [1], we used the simple static Born approximation. Here, we are interested in a fully selfconsistent inclusion of dynamical screening, so the appropriate choice is the random phase approximation (RPA). 


\subsection{Random phase approximation}

Starting from the familiar expression in coordinate representation, application of the gaugeinvariant Fourier transform (31) straightforwardly leads to the following gauge-invariant result

$$
\Sigma_{a}^{\gtrless}\left(\mathbf{k} ; t, t^{\prime}\right)=i \hbar \int \frac{d \mathbf{q}}{(2 \pi \hbar)^{3}} g_{a}^{\gtrless}\left(\mathbf{k}-\mathbf{q} ; t, t^{\prime}\right) V_{a a}^{s \gtrless}\left(\mathbf{q} ; t, t^{\prime}\right),
$$

which transforms the collision integral of Eq.(51) into

$$
\begin{aligned}
I_{a}\left(\mathbf{k}_{a}, t\right) & =-2 \operatorname{Re} \int \frac{d \mathbf{q}}{(2 \pi \hbar)^{3}} \int_{t_{0}}^{t} d \bar{t}\left\{g_{a}^{>}\left[\mathbf{k}_{a}-\mathbf{q}+\mathbf{K}_{a}^{A}(t, \bar{t}) ; t, \bar{t}\right]\right. \\
& \left.\times i \hbar V_{a a}^{s>}(\mathbf{q} ; t, \bar{t}) g_{a}^{<}\left[\mathbf{k}_{a}+\mathbf{K}_{a}^{A}(t, \bar{t}) ; \bar{t}, t\right]-[>\longleftrightarrow<]\right\}
\end{aligned}
$$

In Eq. (52) we introduced the correlation functions of the screened potential (plasmon correlation functions) $V_{s}^{>}, V_{s}^{<}$, which contain the whole screening problem and are directly related to the correlation function of the longitudinal field fluctuations (microfield fluctuations) 53

$$
\frac{e_{a}^{2} \hbar^{2}}{q^{2}} \overline{\left\langle\delta E(t) \delta E\left(t^{\prime}\right)\right\rangle_{\mathbf{q}}}=\frac{1}{2}\left[i \hbar V_{a a}^{s>}\left(\mathbf{q} ; t, t^{\prime}\right)+i \hbar V_{a a}^{s<}\left(\mathbf{q} ; t, t^{\prime}\right)\right]
$$

While in the classical case, the contributions from $V^{>}$and $V^{<}$are equal, in the quantum case a symmetrization is useful which is indicated by the bar over the fluctuation term. $V^{s>}$ and $V^{s<}$ can be related to the retarded and advanced screened potentials via the optical theorem

$$
V_{a b}^{s \gtrless}\left(\mathbf{q} ; t_{1}, t_{2}\right)=\sum_{c} \int_{t_{0}}^{t_{1}} d t_{3} \int_{t_{0}}^{t_{2}} d t_{4} V_{a c}^{s R}\left(\mathbf{q} ; t_{1}, t_{3}\right) \Pi_{c c}^{\gtrless}\left(\mathbf{q} ; t_{3}, t_{4}\right) V_{c b}^{s A}\left(\mathbf{q} ; t_{4}, t_{2}\right),
$$

where $V_{s}^{R}$ and $V_{s}^{A}$ obey the following equation of motion (Dyson equation)

$$
V_{a b}^{s R / A}\left(\mathbf{q} ; t, t^{\prime}\right)=V_{a b}(\mathbf{q}) \delta\left(t-t^{\prime}\right)+\sum_{c} V_{a c}(\mathbf{q}) \int_{t^{\prime}}^{t} d \bar{t} \Pi_{c c}^{R / A}(\mathbf{q} ; t, \bar{t}) V_{c b}^{s R / A}\left(\mathbf{q} ; \bar{t}, t^{\prime}\right),
$$

and $V^{s R / A}$ are related to the nonequilibrium inverse dielectric function according to

$$
V_{a b}^{s R / A}\left(\mathbf{q} ; t, t^{\prime}\right)=V_{a b}(\mathbf{q})\left[\epsilon^{R / A}\left(\mathbf{q} ; t, t^{\prime}\right)\right]^{-1} .
$$

In the above equations, $V_{a b}(\mathbf{q})$ is the bare Coulomb potential (13) and $\Pi^{R / A}$ the retarded and advanced longitudinal polarization functions (plasmon selfenergies). To close this system of equations, the polarization functions have to be expressed in terms of the particle correlation functions for which the simplest approximation is provided by the RPA,

$$
\begin{aligned}
\Pi_{b b}^{\gtrless}\left(\mathbf{q} ; t_{1}, t_{2}\right) & =-i \hbar \int \frac{d^{3} \mathbf{k}_{b}}{(2 \pi \hbar)^{3}} g_{b}^{\gtrless}\left(\mathbf{k}_{b}+\mathbf{q} ; t_{1}, t_{2}\right) g_{b}^{\lessgtr}\left(\mathbf{k}_{b} ; t_{2}, t_{1}\right), \\
\Pi_{b b}^{R}\left(\mathbf{q} ; t, t^{\prime}\right) & =\Theta\left(t-t^{\prime}\right)\left\{\Pi_{b b}^{>}\left(\mathbf{q} ; t, t^{\prime}\right)-\Pi_{b b}^{<}\left(\mathbf{q} ; t, t^{\prime}\right)\right\},
\end{aligned}
$$

and $\Pi^{A}$ follows from the relation $\Pi_{b b}^{A}\left(\mathbf{q} ; t, t^{\prime}\right)=\left[\Pi_{b b}^{R}\left(\mathbf{q} ; t^{\prime}, t\right)\right]^{*}$. This set of equations completely defines the non-Markovian polarization approximation (RPA) for a quantum plasma in a strong transverse field. 


\subsection{Application of the gauge-invariant GKBA}

What is left to obtain a closed expression for the collision integral in equation (51) is to apply the gauge-invariant GKBA (50) together with the free-particle approximation (43) to all two-time functions. This leads to the following results for the optical theorem:

$$
\begin{aligned}
V_{a a}^{s \gtrless}\left(\mathbf{q} ; t_{1}, t_{2}\right)= & -\frac{i}{\hbar} \sum_{b} \int \frac{d^{3} k}{\left(2 \pi \hbar^{3}\right)} \int_{t_{0}}^{t_{1}} d t_{3} \int_{t_{0}}^{t_{2}} d t_{4} V_{a b}^{s R}\left(\mathbf{q} ; t_{1}, t_{3}\right) V_{b a}^{s A}\left(\mathbf{q} ; t_{4}, t_{2}\right) \\
\times & \left\{\Theta\left(t_{3}-t_{4}\right) e^{-\frac{i}{\hbar}\left[\left(\epsilon_{\mathbf{k}+\mathbf{q}}^{b}-\epsilon_{\mathbf{k}}^{b}\right)\left(t_{3}-t_{4}\right)-\mathbf{q} \mathbf{R}_{b}\left(t_{3}, t_{4}\right)\right]}\right. \\
& \times f_{b}^{\gtrless}\left[\mathbf{k}+\mathbf{q}+\mathbf{Q}_{b}\left(t_{3}, t_{4}\right) ; t_{4}\right] f_{b}^{\lessgtr}\left[\mathbf{k}+\mathbf{Q}_{b}\left(t_{3}, t_{4}\right) ; t_{4}\right] \\
& +\Theta\left(t_{4}-t_{3}\right) e^{-\frac{i}{\hbar}\left[\left(\epsilon_{\mathbf{k}+\mathbf{q}}^{b}-\epsilon_{\mathbf{k}}^{b}\right)\left(t_{3}-t_{4}\right)+\mathbf{q} \mathbf{R}_{b}\left(t_{4}, t_{3}\right)\right]} \\
& \left.\times f_{b}^{\gtrless}\left[\mathbf{k}+\mathbf{q}+\mathbf{Q}_{b}\left(t_{4}, t_{3}\right) ; t_{3}\right] f_{b}^{\lessgtr}\left[\mathbf{k}+\mathbf{Q}_{b}\left(t_{4}, t_{3}\right) ; t_{3}\right]\right\} .
\end{aligned}
$$

Similarly, the result for $\Pi^{R}$ can be transformed to

$$
\begin{aligned}
\Pi_{a a}^{R}\left(\mathbf{q} ; t, t^{\prime}\right) & =-\frac{i}{\hbar} \Theta\left(t-t^{\prime}\right) e^{\frac{i}{\hbar} \mathbf{q} \mathbf{R}_{a}\left(t, t^{\prime}\right)} \int \frac{d^{3} k}{(2 \pi \hbar)^{3}} e^{-\frac{i}{\hbar}\left(\epsilon_{\mathbf{k}+\mathbf{q}}^{a}-\epsilon_{\mathbf{k}}^{a}\right)\left(t-t^{\prime}\right)} \\
& \times\left\{f_{a}\left[\mathbf{k}+\mathbf{Q}_{a}\left(t, t^{\prime}\right) ; t^{\prime}\right]-f_{a}\left[\mathbf{k}+\mathbf{q}+\mathbf{Q}_{a}\left(t, t^{\prime}\right) ; t^{\prime}\right]\right\}
\end{aligned}
$$

where the momentum shift $\mathbf{Q}_{a}$ and field-induced displacement $\mathbf{R}_{a}$ were defined above in Eqs. (38) and Eqs. (39), respectively. In the absence of the electromagnetic field, $\left(\mathbf{Q}_{a} \rightarrow\right.$ $0, \mathbf{R}_{a} \rightarrow 0$ ), Eq. (61) reduces to the well-known nonequilibrium RPA-polarization function. The effect of the field is two-fold: first, it introduces an additional retardation $\mathbf{Q}_{a}$ in the distributions (intra-collisional field effect) and second, it leads to a modification of the one-particle energies in the exponent given by $\mathbf{R}_{a}$ which we discussed in detail in ref. [1].

We now can transform the collision integral Eq. (53) by applying the GKBA (50) to $g^{>}, g^{<}$, and using the result for $V^{s>}$ and $V^{s<}$, Eq. (60). After straightforward calculations, we obtain the final result

$$
\begin{aligned}
I_{a}\left(\mathbf{k}_{a}, t\right)= & -\frac{2}{\hbar^{2}} \sum_{b} \operatorname{Re} \int \frac{d \mathbf{k}_{b} d \overline{\mathbf{k}}_{a} d \overline{\mathbf{k}}_{b}}{(2 \pi \hbar)^{6}} \delta\left(\mathbf{k}_{a}+\mathbf{k}_{b}-\overline{\mathbf{k}}_{a}-\overline{\mathbf{k}}_{b}\right) \int_{t_{0}}^{t} d \bar{t} \\
\times & \left\{e^{-\frac{i}{\hbar}\left[\left(\bar{\epsilon}_{a}-\epsilon_{a}\right)(t-\bar{t})+\left(\mathbf{k}_{a}-\overline{\mathbf{k}}_{a}\right) \mathbf{R}_{a}(t, \bar{t})\right]} f_{a}^{>}\left[\overline{\mathbf{k}}_{a}+\mathbf{Q}_{a}(t, \bar{t}) ; \bar{t}\right] f_{a}^{<}\left[\mathbf{k}_{a}+\mathbf{Q}_{a}(t, \bar{t}) ; \bar{t}\right]\right. \\
\times & \int_{t_{0}}^{t} d t_{3} \int_{t_{0}}^{\bar{t}} d t_{4} V^{s R}\left(\mathbf{k}_{a}-\overline{\mathbf{k}}_{a} ; t_{1}, t_{3}\right) V^{s A}\left(\mathbf{k}_{a}-\overline{\mathbf{k}}_{a} ; t_{4}, t_{2}\right) \times \\
& {\left[\Theta\left(t_{3}-t_{4}\right) e^{-\frac{i}{\hbar}\left[\left(\bar{\epsilon}_{b}-\epsilon_{b}\right)\left(t_{3}-t_{4}\right)+\left(\mathbf{k}_{b}-\overline{\mathbf{k}}_{b}\right) \mathbf{R}_{b}\left(t_{3}, t_{4}\right)\right]}\right.} \\
& \times f_{b}^{>}\left[\overline{\mathbf{k}}_{b}+\mathbf{Q}_{b}\left(t_{3}, t_{4}\right) ; t_{4}\right] f_{b}^{<}\left[\mathbf{k}_{b}+\mathbf{Q}_{b}\left(t_{3}, t_{4}\right) ; t_{4}\right] \\
& +\Theta\left(t_{4}-t_{3}\right) e^{-\frac{i}{\hbar}\left[\left(\bar{\epsilon}_{b}-\epsilon_{b}\right)\left(t_{3}-t_{4}\right)-\left(\mathbf{k}_{b}-\overline{\mathbf{k}}_{b}\right) \mathbf{R}_{b}\left(t_{4}, t_{3}\right)\right]} \\
& \left.\left.\times f_{b}^{>}\left[\overline{\mathbf{k}}_{b}+\mathbf{Q}_{b}\left(t_{4}, t_{3}\right) ; t_{3}\right] f_{b}^{<}\left[\mathbf{k}_{b}+\mathbf{Q}_{b}\left(t_{4}, t_{3}\right) ; t_{3}\right]\right] \quad[>\longleftrightarrow<]\right\} .(62) \quad[><
\end{aligned}
$$

This is the general non-Markovian collision integral for a homogeneous weakly coupled $d y$ namically screened plasma in an electromagnetic field. It is a generalization of numerous results which were previously obtained by various authors, including our static screening 
result for strong time-dependent fields [1] and the result of Silin and Uryupin [35], the RPA result for a static field of Morawetz [40] and the field-free RPA results of Kuznetsov 38] and Haug and Ell [39]. Furthermore, it generalizes previous results obtained for classical plasmas by Silin, Oberman et al., Klimontovich and others [23, 27, 14]. In particular, as we will see below, the classical dynamical screening result of Klimontovich and Puchkov [29] is straightforwardly recovered from the collision integral (62). This collision integral is the basis for computing electron-ion collision frequencies, plasma heating inverse bremsstrahlung effects etc., thereby fully taking into account dynamical screening, plasma instabilities and anomalous transport

Despite the complicated structure of the integral (62), a direct numerical integration of the kinetic equation (51) appears to be within reach, as recently solutions of non-Markovian RPA-type equations (without longitudinal field) for semiconductors have been reported [20, 54, 55]. On the other hand, to gain deeper insight in the physical processes contained in the collision term (62), it is useful to consider analytical simplifications.

\subsection{High frequency fields. Silin ansatz}

If the collision frequency is low compared to the oscillation frequency of the field, i.e. if the parameter $\delta \ll 1$, one may follow an idea of Silin [25] and solve the kinetic equation (51) with a perturbation ansatz $f_{a}=f_{a}^{0}+f_{a}^{1}$ where $f_{a}^{0}$ obeys the collisionless equation

$$
\begin{array}{r}
\frac{\partial}{\partial t} f_{a}^{0}(\mathbf{k}, t)+e_{a} \mathbf{E}(t) \cdot \nabla_{\mathbf{k}} f_{a}^{0}(\mathbf{k}, t)=0 \\
\text { with the solution } \quad f_{a}^{0}(\mathbf{k}, t)=f_{a 0}\left[\mathbf{k}+\frac{e_{a}}{c} \mathbf{A}(t)\right],
\end{array}
$$

where $f_{a 0}$ is an arbitrary function depending on the initial conditions. The equation for $f_{a}^{1}$ reads

$$
\begin{array}{r}
\frac{\partial}{\partial t} f_{a}^{1}(\mathbf{k}, t)+e_{a} \mathbf{E}(t) \cdot \nabla_{\mathbf{k}} f_{a}^{1}(\mathbf{k}, t)=I_{a}^{1}(\mathbf{k}, t) \\
\text { where } \quad I_{a}^{1}(\mathbf{k}, t)=I_{a}\left\{f_{a} \rightarrow f_{a 0}\left[\mathbf{k}+\frac{e_{a}}{c} \mathbf{A}(t)\right]\right\} .
\end{array}
$$

With this scheme, there follow essential simplifications of the above results because it effectively eliminates the time retardation of the distribution functions in the collision integrals [56]. Indeed, one easily verifies that the arguments of the distributions which appear in the formulas above, now become

$$
f_{a}\left[\mathbf{k}+\mathbf{Q}_{a}\left(t, t^{\prime}\right) ; t^{\prime}\right] \longrightarrow f_{a 0}\left[\mathbf{k}+\frac{e_{a}}{c} \mathbf{A}(t)\right],
$$

and do not depend on the time $t^{\prime}$ anymore. This simplification allows to compute the transport, screening and fluctuation properties quite efficiently.

We first consider the modification of the longitudinal polarization $\Pi^{R}$. Straightforward transformations of Eq. (61) including a change of the momentum integration variable lead to

$$
\begin{aligned}
\Pi_{a a}^{R}\left(\mathbf{q} ; t, t^{\prime}\right) & =e^{i \frac{e_{a \mathbf{q}}}{m_{a} \hbar \hbar} \int_{t^{\prime}}^{t} d \overline{\mathbf{t}} \mathbf{A}(\bar{t})} \cdot \Pi_{a a, 0}^{R}\left(\mathbf{q} ; t-t^{\prime}\right) \\
\text { whith } \quad \Pi_{a a, 0}^{R}(\mathbf{q} ; \tau) & =-\frac{i}{\hbar} \Theta(\tau) \int \frac{d^{3} k}{(2 \pi \hbar)^{3}} e^{-\frac{i}{\hbar}\left(\epsilon_{\mathbf{k}+\mathbf{q}}^{a}-\epsilon_{\mathbf{k}}^{a}\right) \tau}\left\{f_{a}(\mathbf{k})-f_{a}(\mathbf{k}+\mathbf{q})\right\} .
\end{aligned}
$$

Using this result and the adiabatic approximation (neglecting the ion contribution to the polarization), we obtain from the Dyson equation, Eq. (56), for the retarded screened potential

$$
V_{a b}^{R}\left(\mathbf{q} ; t, t^{\prime}\right)=e^{i \frac{e_{a} \mathbf{q}}{m_{a} c \hbar} \int_{t^{\prime}}^{t} d \bar{t} \mathbf{A}(\bar{t})} \cdot V_{a b, 0}^{R}\left(\mathbf{q} ; t-t^{\prime}\right)
$$


where again, $V_{a b, 0}^{R}$ denotes the screened potential in the zero field limit and without retardation in the distribution functions. Simplifications are possible also for the collision integrals. In particular, we obtain for the electron-ion scattering term,

$$
\begin{aligned}
& I_{e i}\left(\mathbf{k}-\frac{e_{a}}{c} \mathbf{A}(t)\right)=\frac{2 n_{i}}{\hbar^{2}} \operatorname{Re} \int \frac{d^{3} q}{(2 \pi \hbar)^{3}}\left|V_{e i}(q)\right|^{2} \int_{-\infty}^{0} d \tau e^{-\frac{i}{\hbar}\left[\epsilon_{\mathbf{k}}^{e}-\epsilon_{\mathbf{k}-\mathbf{q}}^{e}\right] \tau}
\end{aligned}
$$

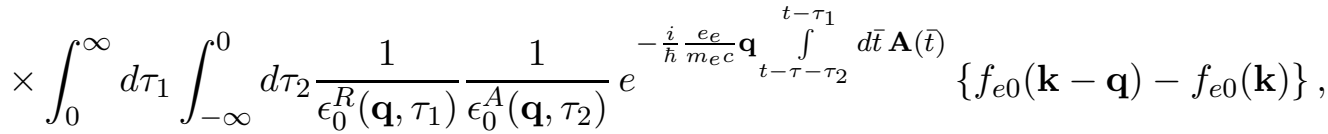

where $1 / \epsilon_{0}^{R / A}$ are the field-free inverse dielectric functions. Eq. (69) is the generalization of Klimontovich's result [14] to quantum plasmas and.

Finally, we consider the simplifications introduced by the ansatz $(\sqrt{64})$ to the field fluctuations. The quantity of central importance is the correlation function of the temporal and spatial microfield fluctuations $\langle\delta E \delta E\rangle$, Eq. (54). A lengthy but straightforward calculation leads to the following result

$$
\begin{aligned}
& \overline{\left\langle\delta \mathbf{E}\left(t_{1}\right) \delta \mathbf{E}\left(t_{2}\right)\right\rangle_{\mathbf{q}}}=\frac{(4 \pi)^{2}}{2 q^{2}} e^{i \frac{e_{a} \mathbf{q}}{m_{a} c \hbar} \int_{t_{2}}^{t_{1}} d \bar{t} \mathbf{A}(\bar{t})} \int \frac{d^{3} k}{(2 \pi \hbar)^{3}} \\
& \left\{e_{e}^{2} \frac{e^{-\frac{i}{\hbar}\left[\epsilon_{\mathbf{k}+\mathbf{q}}^{e}-\epsilon_{\mathbf{k}}^{e}\right]\left(t_{1}-t_{2}\right)}}{\left|\epsilon_{0}\left(\mathbf{q}, \epsilon_{\mathbf{k}+\mathbf{q}}^{e}-\epsilon_{\mathbf{k}}^{e}\right)\right|^{2}}\left\{f_{e 0}(\mathbf{k}+\mathbf{q})\left[1-f_{e 0}(\mathbf{k})\right]+f_{e 0}(\mathbf{k})\left[1-f_{e 0}(\mathbf{k}+\mathbf{q})\right]\right\}\right. \\
& +e_{i}^{2} e^{-\frac{i}{\hbar}\left[\epsilon_{\mathbf{k}+\mathbf{q}}^{i}-\epsilon_{\mathbf{k}}^{i}\right]\left(t_{1}-t_{2}\right)} \int_{0}^{\infty} d \tau_{1} \frac{e^{-\frac{i}{\hbar}\left[\epsilon_{\mathbf{k}+\mathbf{q}}^{i}-\epsilon_{\mathbf{k}}^{i}\right] \tau_{1}}}{\epsilon_{0}^{R}\left(\mathbf{q}, \tau_{1}\right)} \\
& \left.\quad \times \int_{-\infty}^{0} d \tau_{2} \frac{e^{\frac{i}{\hbar}\left[\epsilon_{\mathbf{k}+\mathbf{q}}^{i}-\epsilon_{\mathbf{k}}^{i}\right] \tau_{2}}}{\epsilon_{0}^{A}\left(\mathbf{q}, \tau_{2}\right)} e^{-\frac{i}{\hbar}\left(\frac{e e}{m_{e}}-\frac{e_{i}}{m_{i}}\right) \mathbf{q}_{t_{2}-\tau_{2}}^{t_{1}-\tau_{1}} d \bar{t} \frac{\mathbf{A}}{c}(\bar{t})}\left[f_{i 0}(\mathbf{k}+\mathbf{q})+f_{i 0}(\mathbf{k})\right]\right\},
\end{aligned}
$$

where the first term in parantheses (second line) is the electron contribution, and the second (third and fourth line) results from the ions. Again, this is a generalization of Klimontovich's remarkable result [14 who considered the classical limit and the equal time fluctuations, $t_{1}=t_{2}$. Our result fully includes the two-time fluctuations which are directly measurable quantities. From the above fluctuation spectrum, all major observables of dense quantum plasmas in a strong laser field can be computed. The corresponding analysis will be presented in a forthcoming paper.

\section{Discussion}

In this paper, we have presented a gauge-invariant derivation of the quantum kinetic equation for dense plasmas in a laser field. Our main result, Eq. (62), generalizes previous work to quantum systems. This equation can be used to calculate the transport properties of a dense plasma in a laser field on arbitrary time scales, i.e., over the whole frequency range. The use of the random phase approximation allows for a highly consistent treatment of the combined effect of internal longitudinal fields (dynamical screening) and transverse electromagnetic fields, including intense laser pulses. In particular, it allows to investigate the influence of the electromagnetic field on the two-particle scattering process and the screening properties of the plasma and on the screening buildup in the presence of a strong field.

Besides, the presented gauge-invariant approach is completely general and can be extended straightforwardly to more complex situations, including strong coupling effects, bound states, impact and field ionization. Moreover, it can be directly generalized to relativistic systems und ultra-intense fields. 


\section{Acknowledgments}

It is a great pleasure to dedicate this paper to Yuri Lvovich Klimontovich on his seventyfith birthday. All of us have, at different times, come in contact with the remarkable papers and monographs of Klimontovich. We have very much enjoyed countless discussions with him as well as rewarding scientific collaborations on problems of kinetic theory of nonideal gases and plasmas in general, in particular on dielectric properties [57], kinetic equations with bound states [58, 59], foundation of reaction-diffusion equations for nonideal plasmas [60], selforganization and structure formation [61] and many others.

The authors acknowledge stimulating discussions with G. Kalman, P. Mulser and R. Sauerbrey. This work is supported by the Deutsche Forschungsgemeinschaft (Schwerpunkt "Wechselwirkung intensiver Laserfelder mit Materie"), and by the European Commission through the TMR Network SILASI.

\section{References}

[1] D. Kremp, Th. Bornath, M. Bonitz, and M. Schlanges, Phys. Rev. E (1999)

[2] for a recent overview, see e.g. M.D. Perry, and G. Mourou, Science 264 (1994) 917

[3] M. Tabak et al., Phys. Plasmas 1 (1994) 1626

[4] W. Theobald, R. Hässner, C. Wülker, and R. Sauerbrey, Phys. Rev. Lett. 77 (1996) 298

[5] I. Prigogine, Nonequilibrium Statistical Mechanics, Interscience, New York, 1963.

[6] R. Zwanzig, J. Chem. Phys. 33 (1960) 1338; Physica 30 (1964) 1109.

[7] L.P. Kadanoff and G. Baym, Quantum Statistical Mechanics, Addison-Wesley Publ. Co. Inc., 2nd ed., 1989.

[8] G. Baym, and L.P. Kadanoff, Phys. Rev. 124 (1961) 287

[9] R. Balescu, Statistical Mechanics of Charged Particles, Interscience Publishers, 1963

[10] R. Balescu, Equilibrium and Nonequilibrium Statistical Mechanics, Wiley, New York, 1975.

[11] V.P. Silin Kinetic equations for a gas of charged particles, supplement to the russ. edition of ref. 9], Nauka, Moscow 1967

[12] Yu.L. Klimontovich, Statistical Theory of Nonequilibrium Processes (russ.), Izdat. MGU 1964

[13] Yu. L. Klimontovich, and W. Ebeling, ZhETF 63 (1972) 905 [Sov. Phys. JETP 36 (1973) 476]

[14] Yu.L. Klimontovich, Kinetic Theory of Nonideal Gases and Nonideal Plasmas, Nauka, Moscow 1975 (russ.), Engl. transl.: Pergamon Press, Oxford 1982.

[15] M. Bonitz, and D. Kremp, Phys. Lett. A 212 (1996) 83

[16] D. Kremp, M. Bonitz, W.D. Kraeft, and M. Schlanges, Ann. Phys. (N.Y.) 258 (1997) 320

[17] D. Kremp, Th. Bornath, M. Bonitz, and M. Schlanges, Physica B 228 (1996) 72

[18] Th. Bornath, D. Kremp, W.D. Kraeft, and M. Schlanges, Phys. Rev. E 54 (1996) 3274

[19] M. Bonitz, D. Kremp, D.C. Scott, R. Binder, W.D. Kraeft, and H.S. Köhler, J. Phys.: Condensed Matter 8 (1996) 6057

[20] L. Banyai, Q.T. Vu, B. Mieck, and H. Haug, Phys. Rev. Lett. 81 (1998) 882

[21] H. Haug, A.P. Jauho, Quantum Kinetics in Transport and Optics of Semiconductors, Springer-Verlag, Heidelberg, New York, 1996. 
[22] M. Bonitz, Quantum Kinetic Theory, B.G. Teubner, Stuttgart, Leipzig 1998.

[23] For an overview on early work on kinetic equations for plasmas, cf. V.P. Silin, ZhETF 38 (1960) 1771.

[24] V.P. Silin, ZhETF 41 (1961) 861.

[25] V.P. Silin, ZhETF 47 (1964) 2254.

[26] J. Dawson, and C. Oberman, Phys. Fluids 5 (1962) 517

[27] C. Oberman, A. Ron, and J. Dawson, Phys. Fluids 5 (1962) 1514.

[28] D.F. DuBois, V. Gilinsky, and Margaret G. Kivelson, Phys. Rev. 129 (1963) 2376

[29] Yu. L. Klimontovich, and V.A. Puchkov, ZhETF 67 (1974) 556

[30] Yu. L. Klimontovich, ZhETF 33 (1957) 982

[31] Yu.L. Klimontovich, Kinetic Theory of Electromagnetic Processes, Nauka, Moscow 1980 (russ.), Engl. transl.: Springer 1982

[32] C.D. Decker, W.B. Mori, J.M. Dawson, and T. Katsuoleas, Phys. Plasmas 1 (1994) 4043

[33] P. Mulser, and A. Saemann, Contr. Plasma Phys. 37 (1997) 211

[34] P. Mulser, F. Cornolti, E. Bésuelle, and R. Schneider, submitted for publication

[35] V.P. Silin, and S.A. Uryupin, ZhETF 81 (1981) 910

[36] K. Morawetz, and D. Kremp, Phys. Lett. A 173 (1993) 317.

[37] K. Morawetz, M. Schlanges, D. Kremp, Phys. Rev. E 48 (1993) 2980.

[38] A.V. Kuznetsov, Phys. Rev. B 44 (1991) 8721

[39] H. Haug, and C. Ell, Phys. Rev. B 46 (1992) 2126

[40] K. Morawetz, Phys. Rev. E 50 (1994) 4625

[41] Yu. L. Klimontovich, and V.P. Silin, Doklady Akad. Nauk 82 (1952) 361

[42] Yu. L. Klimontovich, and V.P. Silin, ZhETF 23 (1952) 151

[43] Yu. L. Klimontovich, and V.P. Silin, For an overview on early work on kinetic equations for plasmas, cf. Uspekhi Fiz. Nauk 70 (1960) 247 [Sov. Phys. Uspekhi 3 (1961) 84]

[44] D.F. DuBois, In "Lectures in Theoretical Physics", vol. IX C, edited by W.E. Brittin, A.O. Barut, and M. Guenin, p. 469

[45] B. Bezzerides, and D.F. DuBois, Ann. Phys. (N.Y.) 70 (1972) 10

[46] It is interesting to note that Klimontovich's phase space densities $N$ [30] are essentially the classical limit of the field operators. Therefore, his kinetic theory is of similar generality as modern field theory, cf. his text books 12, 14, 31.

[47] D. Kremp, M. Schlanges, Th. Bornath, J. Stat. Phys. 41 (1985) 661.

[48] D. Semkat, D. Kremp, and M. Bonitz, Phys. Rev. E 59 (1999) 1557

[49] P. Lipavský, V. Špička, and B. Velický, Phys. Rev.B 34 (1986) 6933

[50] S. Fujita, Introduction to Nonequilibrium Quantum Statistical Mechanics, W.B. Saunders Company, Philadelphia, London, 1966.

[51] D.M. Volkov, Z. Phys. 94 (1934) 125 
[52] A.-P. Jauho, and K. Johnsen, Phys. Rev. Lett. 76 (1996) 4576

[53] M. Schlanges, and Th. Bornath, Wiss. Zeitschr. Univ. Rostock Jahrg. 36 (1987) 65

[54] N. Kwong, and M. Bonitz, unpublished

[55] U. Moldzio, priv. communication (1999)

[56] This ansatz is analogous to the zeroth order retardation ansatz of non-Markovian field-free kinetic equations, e.g. [22].

[57] Yu.L. Klimontovich, and W.D. Kraeft, Teplofiz. Vys. Temp. (russ.) 12 (1974) 239

[58] Yu.L. Klimontovich, and D. Kremp, Physica A 109 (1981) 517

[59] Yu.L. Klimontovich, D. Kremp, and W.D. Kraeft, Advances of Chem. Phys. 58 (1987) 175

[60] Yu.L. Klimontovich, M. Schlanges, and Th. Bornath, Contrib. Plasma Phys. 30 (1990) 349

[61] Yu.L. Klimontovich, and M. Bonitz, Z. Phys. B 70 (1988) 241

Received 